intrinsically deterministic - because of the high efficiency of the homodyne measurement and the low light-absorption losses, the teleportation protocol succeeds at every attempt. Similar protocols could be applied to a wide range of quantum systems, for example, to teleport motional states of the micromechanical harmonic oscillators, or spin states of a cold ensemble of atoms or molecules coupled to microwave field in a high finesse resonator.

Dzmitry Matsukevich is at the Centre for Quantum Technologies and Department of Physics,

National University of Singapore,

117543 Singapore.

e-mail:phymd@nus.edu.sg
References

1. Bouwmeester, D. et al. Nature 390, 575-579 (1997).

$\square$ 2. Furusawa, A. et al. Science 282, 706-709 (1998).

3. Olmschenk, S. et al. Science 323, 486 (2009).

4. Nölleke, C. et al. Phys. Rev. Lett. 110, 140403 (2013).

5. Bao, X-H. et al. Proc. Natl Acad. Sci. USA 109, 20347-20351 (2012).

6. Krauter, H. et al. Nature Phys. 9, 400-404 (2013).

Published online: 2 June 2013

\title{
HYDRODYNAMICS
}

\section{Wake up}

Google Earth as a hydrodynamics lab? Indeed - studying its satellite photos of moving ships and their trails has enabled Marc Rabaud and Frédéric Moisy to propose a neat, general theory for the dependence of a boat's wake angle on its speed (Phys. Rev. Lett. 110, 214503; 2013).

A physical explanation for the phenomenon of boat wakes, and for the value of the wake angle, was originally given by William Thomson - better known as Lord Kelvin. He found that the faster the speed of the ship, the faster the wake broadened laterally. Consequently, the wake angle has a constant value of about $19.5^{\circ}$. However, it was later noted that contrary to Kelvin's finding - smaller wake angles also occur.

By examining a selection of aerial photos featuring different types of

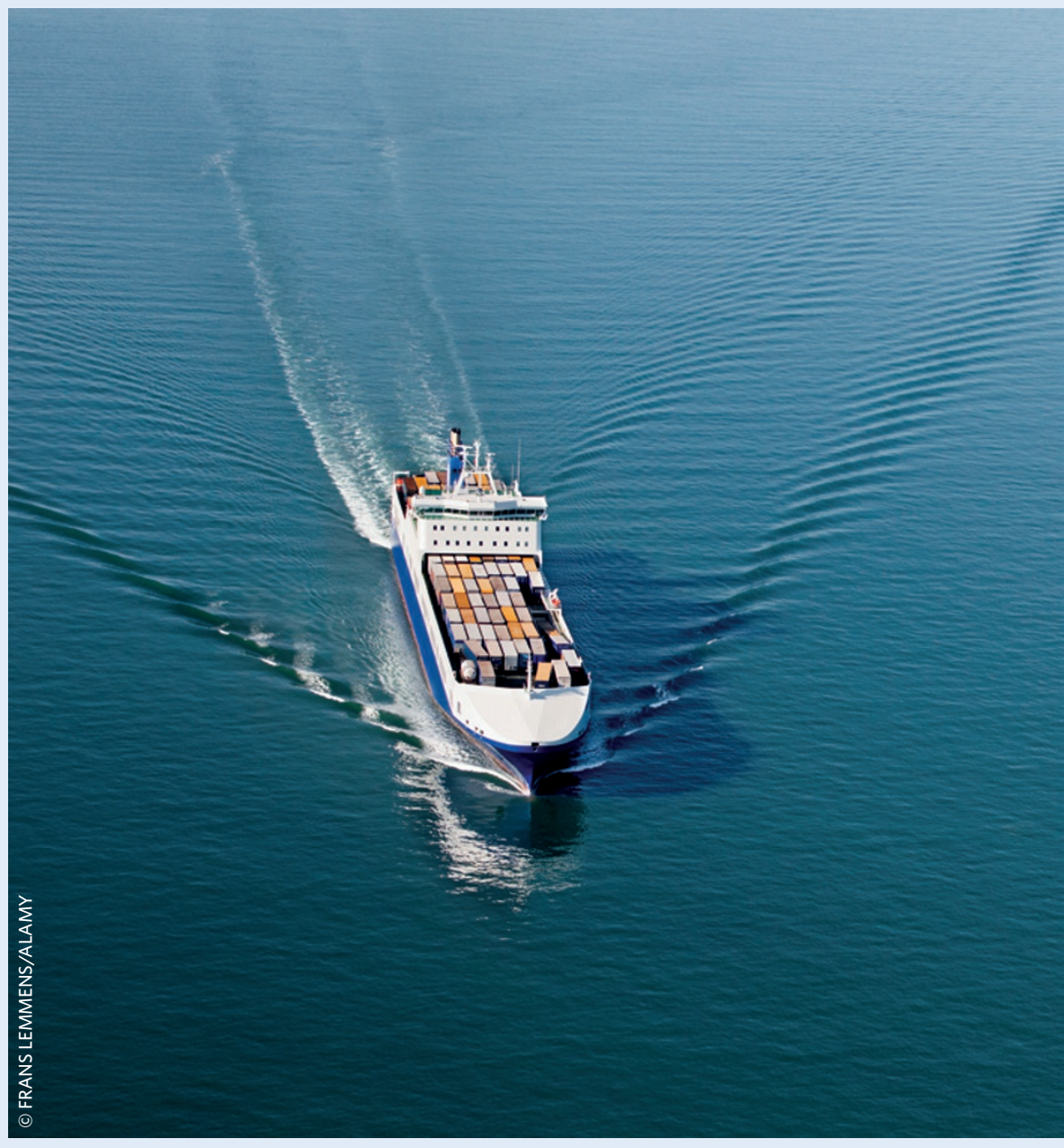

moving vessels, Rabaud and Moisy first confirmed the existence of narrower wakes. (The Google Earth images were carefully chosen: regions close to a harbour give the best graphical resolution, and ships have to be moving in a straight line.) They measured the wake angle and length of each ship, and estimated its velocity using the wavelength of the waves in the arms of the ship's wake. Plotting the wake angle versus the so-called Froude number, $\mathrm{Fr}-$ a dimensionless quantity proportional to the boat's speed and inversely proportional to the square root of its length - uncovers two regimes: for small Fr the wake angle is constant; but for large values, the wake angle decays with increasing Fr.

These observations can be understood by realizing that a moving boat cannot excite waves that have larger wavelengths than its own length. Taking this into account, the authors recover the Kelvin angle of $19.5^{\circ}$ for $\mathrm{Fr}<0.5$, and the decay law above $\mathrm{Fr}=0.5$ - the wake angle is then inversely proportional to Fr, that is, inversely proportional to the ship's velocity. The narrowing of the wake with increasing speed is analogous to the tapering of the Mach cone of accelerating supersonic aircraft.

The data points do not, however, lie perfectly on the theoretical curve. Rabaud and Moisy suggest that this is due to uncertainties in measuring the ship lengths (the actual waterline may be smaller than the contour seen from above) and wake wavelengths that are required for determining ship speeds. So, although it's an original approach to hydrodynamics, the Google Earth laboratory - like any other - isn't error-free. 